Gut and Liver, Vol. 9, No. 5, September 2015, pp. 601-606

\title{
Infliximab versus Cyclosporine Treatment for Severe Corticosteroid- Refractory Ulcerative Colitis: A Korean, Retrospective, Single Center Study
}

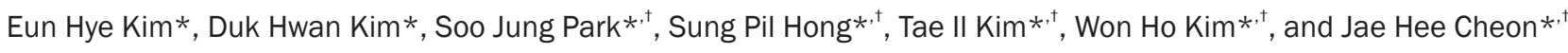 \\ ${ }^{*}$ Department of Internal Medicine and ${ }^{\dagger}$ Institute of Gastroenterology, Yonsei University College of Medicine, Seoul, Korea
}

\section{See editorial on page 567.}

Background/Aims: In patients with corticosteroid-refractory ulcerative colitis (UC), cyclosporine or infliximab may be added to the treatment regimen to induce remission. Here, we aimed to compare the efficacy of cyclosporine and infliximab. Methods: Between January 1995 and May 2012, the medical records of 43 patients with corticosteroid-refractory UC who received either infliximab or cyclosporine as a rescue therapy at a tertiary care hospital in Korea were reviewed. Results: Among the 43 patients, 10 underwent rescue therapy with cyclosporine and the remaining 33 patients received infliximab. A follow-up of 12 months was completed for all patients. The colectomy rate at 12 months was 30\% and $3 \%$ in the cyclosporine and the infliximab groups, respectively ( $p=0.034)$. However, the Cox proportional hazard model indicated that the treatment of rescue therapy was not an independent associate factor for preventing colectomy $(p=0.164)$. In the subgroup analysis, infliximab with azathioprine was superior to cyclosporine for preventing colectomy (hazard ratio of infliximab with azathioprine compared with cyclosporine only, 0.073; 95\% confidence interval, 0.008 to 0.629). Conclusions: No difference between infliximab and cyclosporine with respect to preventing colectomy was noted. However, infliximab with azathioprine may be more effective than cyclosporine alone for preventing colectomy. (Gut Liver 2015;9:601-606)

Key Words: Colitis, ulcerative; Cyclosporine; Infliximab

\section{INTRODUCTION}

Acute severe ulcerative colitis (UC) is a potentially lethal clinical condition that requires intensive medical treatment and potential colectomy in cases of treatment failure. ${ }^{1,2}$ A recent study showed that acute colitis can occur in up to $25 \%$ of patients with UC. ${ }^{3,4}$ Use of intravenous corticosteroids has been shown to modify the natural history of severe acute relapses, but approximately $30 \%$ to $40 \%$ of patients fail to respond to this intensive treatment. ${ }^{5,6}$ Both infliximab and cyclosporine have been shown to be effective in inducing remission or at least delaying surgery in patients with severe UC that is refractory to intravenous high-dose corticosteroids. ${ }^{1,7-9}$

Cyclosporine is an immunosuppressant that inhibits T-cell mediated production of interleukin 2 (IL-2). ${ }^{10}$ Acute remission rates achieved with the use of cyclosporine have ranged between 63\% and 82\% in previous trials. ${ }^{10-13}$ Although these studies showed some benefits of cyclosporine treatment, there is a significant risk of toxicity that can result in hypertension, seizures, renal impairment, and hypomagnesaemia, which require intensive monitoring. ${ }^{14}$ In the last 10 years, infliximab, which is a monoclonal antibody that binds free and membrane-bound tumor necrosis factor $\alpha$, has been shown to be an effective treatment for severe UC. At a dosage of $5 \mathrm{mg} / \mathrm{kg}$, infliximab can help to reduce the risk of colectomy. ${ }^{15}$

A recent retrospective review investigating outcomes of patients treated with either infliximab or cyclosporine as a rescue therapy for UC requiring hospitalization concluded that there is no difference between the two with respect to induction of clinical remission or avoiding urgent colectomy at 3 months. ${ }^{8,11,16,17}$ Moreover, in a European study ${ }^{17}$ treatment failure was noted in 35 of 58 patients (60\%) given cyclosporine compared with 31 of 57 (54\%) given infliximab ( $p=0.52)$. There was no difference

Correspondence to: Jae Hee Cheon

Department of Internal Medicine, Yonsei University College of Medicine, 50 Yonsei-ro, Seodaemun-gu, Seoul 120-752, Korea

Tel: +82-2-393-6884, Fax: +82-2-2228-1990, E-mail: geniushee@yuhs.ac

Received on March 31, 2014. Revised on May 16, 2014. Accepted on May 21, 2014. Published online December 5, 2014

pISSN 1976-2283 eISSN 2005-1212 http://dx.doi.org/10.5009/gnl14120

(a) This is an Open Access article distributed under the terms of the Creative Commons Attribution Non-Commercial License (http://creativecommons.org/licenses/by-nc/4.0) which permits unrestricted non-commercial use, distribution, and reproduction in any medium, provided the original work is properly cited. 
between the two groups in terms of treatment failure. ${ }^{17}$ Most of these data, however, have been reported in Western populations which have different genetic and ethnic backgrounds than Asian populations. To date, there has been little data on use of these treatments in Asian countries. According to the first data reported in a Korean population that investigated the efficacy of infliximab in patients with UC, the rates of clinical response and remission were $87 \%$ and $45 \%$ after 8 weeks and long-term were $71 \%$ and $62 \%$, respectively. ${ }^{18}$ They concluded that infliximab is effective and safe in the treatment of active UC in Korean patients. The study, however, evaluated the efficacy of infliximab only without group comparison. We, therefore, aimed to compare the efficacy of infliximab with that of cyclosporine in severe steroid-refractory UC patients in Korean population.

\section{MATERIALS AND METHODS}

\section{Patients}

We reviewed records from a series of patients with severe corticosteroid-refractory UC admitted to Severance Hospital between January 1995 and May 2012 and compared outcomes after treatment with cyclosporine or infliximab. The diagnosis of UC was made according to previously established international criteria based on clinical, endoscopic, histopathologic, and radiographic findings. ${ }^{19}$ Intravenous corticosteroid-resistant UC is defined as a lack of response to an adequate dosage of corticosteroids within 5 to 7 days. ${ }^{20}$ Patients were eligible for inclusion in this study if they had been hospitalized between January 1995 and May 2012 for treatment of acute UC that was refractory to intravenous hydrocortisone, and they were subsequently treated with either intravenous cyclosporine or infliximab.

Between 1995 and 2005, all patients seen at our clinic with severe UC refractory to intravenous corticosteroids were treated with cyclosporine at the dosage of $2 \mathrm{mg} / \mathrm{kg}$ daily, with adjustments in dosage based on blood levels of cyclosporine (therapeutic range, 160 to $360 \mu \mathrm{g} / \mathrm{L}$ ). Intravenous cyclosporine was continued until a response was achieved, and then the patient was switched to azathioprine. From 2006 to the present, patients with this condition were treated with infliximab at the dosage of $5 \mathrm{mg} / \mathrm{kg}$ after screening for infection or malignancy. Patients who responded to infliximab without clinical signs of intolerance completed the induction phase with infusions at weeks 2 and 6 followed by scheduled infusions ( $5 \mathrm{mg} / \mathrm{kg}$ every 8 weeks).

In some patients treated with infliximab or cyclosporine, azathioprine was started soon after the last infusion. If previous intolerance or failure of immunomodulators had been reported, maintenance treatment with azathioprine was not performed.

In all patients in whom cyclosporine or infliximab failed, a total colectomy was performed. Cyclosporine or infliximab failure was determined by a physician after a global assessment, and a clinical decision was made regarding whether continued medical treatment or an emergency colectomy was appropriate within 5 to 7 days.

\section{Data collection}

All medical records were retrospectively reviewed. Our team has utilized a specialized medical record form at our inflammatory bowel diseases clinic for the past 20 years. Patients were interviewed by clinical research coordinators and invited to answer a study questionnaire, which addressed data related to UC forms that scored clinical activity. A physician's global assessment was performed for each patient at every outpatient clinic visit or hospitalization. The accumulated data were converted into indices of clinical disease activity for this study. The following information was collected: type of intervention, age, sex, extent of disease, duration of disease prior to admission, length of intravenous corticosteroid treatment prior to intervention, and medications at time of admission. The extent of disease was measured by colonoscopy or sigmoidoscopy based on patients' conditions. Laboratory measurements were also recorded including erythrocyte sedimentation rate, hemoglobin, and albumin at the start of and throughout the course of treatment. The date of colectomy was also recorded for each patient. The C-reactive protein (CRP) level was checked at the start of treatments.

\section{End-points}

The primary end-point was a colectomy-free survival. Secondary end-points were colectomy rates at 3 and 12 months, remission inducing and maintenance rates at 3 and 12 months, length of hospital stay after initiation of either treatment, steroid requirement at 12 months, and adverse events.

\section{Statistical analysis}

Continuous variables including age, duration of disease prior to intervention, and CRP level were summarized as means \pm standard deviations or medians with ranges according to their distribution. Categorical variables including drugs for rescue therapy, disease extension, and number of patients on azathioprine therapy at the time of treatment were summarized as frequencies and percentages. Statistical analysis included Student t-tests, Mann-Whitney U tests, chi-square tests, and Fisher exact tests. Kaplan-Meier plots and log-rank tests were performed for survival analysis of the two treatment groups. A Cox proportional hazards model was proposed in order to adjust for confounding variables. We considered the following possible confounders: age, disease duration, length of treatment with intravenous corticosteroids, use of azathioprine, length of hospitalization, CRP values, disease extension. Variables with significance of 0.2 or less on univariate analysis were included as covariates in a Cox proportional hazards model. If the discrepancy of results between univariate and multivariate analyses was identified, subgroup analysis was performed for evaluating 
Table 1. Baseline Characteristics of Patients with Severe Ulcerative Colitis

\begin{tabular}{lccc}
\hline \multicolumn{1}{c}{ Characteristic } & Cyclosporine $(\mathrm{n}=10)$ & Infliximab (n=33) & $\mathrm{p}$-value \\
\hline Age, yr & $56(22-72)$ & $44(15-71)$ & 0.678 \\
Male sex & $3(30.0)$ & $25(75.8)$ & 0.008 \\
Duration of disease prior to intervention, mo & $121.4(0-216)$ & $76.3(0-192)$ & 0.506 \\
Disease extension & & $12(36.4)$ & \\
Extensive colitis & $8(80.0)$ & $3(9.1)$ \\
Nonextensive colitis & $1(10.0)$ & $16(48.5)$ \\
$\quad$ Left-sided colitis & $1(10.0)$ & $2(6.1)$ \\
$\quad$ Rectosigmoid colitis & 0 & $30.0(28-489)$ \\
$\quad$ Proctitis & $10.9(1.9-48.0)$ & $28(84.8)$ & 0.057 \\
C-reactive protein (mg/L) at the time of treatment & $4(40.0)$ & 0.004 \\
Patients on azathioprine therapy at the time of treatment & &
\end{tabular}

Data are presented as median (range) or number (\%).

${ }^{*} \mathrm{p}$-value between the extensive colitis and nonextensive colitis groups.

Table 2. Colectomy Rates at 3 Months, 12 Months, and at the End of the Follow-Up Period

\begin{tabular}{lccc}
\hline Colectomy rate & Cyclosporine & Infliximab & p-value \\
\hline 3 Months (\%) & $1(10.0)$ & 0 & 0.233 \\
12 Months $(\%)$ & $3(30.0)$ & $1(3.0)$ & 0.034 \\
3 Years $(\%)$ & $6(60.0)$ & $1(3.0)$ & $<0.001$ \\
\hline
\end{tabular}

the reason of discrepancy. A p-value $<0.05$ was regarded as significant for between group comparisons. All statistical analyses were performed using SPSS version 18.0 for Windows (SPSS Inc., Chicago, IL, USA).

\section{RESULTS}

\section{Clinical characteristics of study subjects}

A total of 43 patients were included in the final analysis: 10 in the cyclosporine group and 33 in the infliximab group. Patient characteristics are shown in Table 1 . The median followup period was 119.4 months (range, 22.5 to 207.8 months) in the cyclosporine group and 50.6 months (range, 11.2 to 195.7 months) in the infliximab group. Four patients (40\%) in the cyclosporine group and 28 patients (84.8\%) in the infliximab group were taking azathioprine on admission.

\section{Outcomes}

A 12-month follow-up was completed for all patients. Three months after the acute episode requiring either cyclosporine or infliximab treatment, the colectomy rate was 10\% (1 of 10) in the cyclosporine group and 0\% (0 of 33) in the infliximab group ( $\mathrm{p}=0.233$ ). At 12 months, the colectomy rate increased to $30 \%$ in the cyclosporine group versus 3\% in the infliximab group ( $\mathrm{p}=0.034$ ) (Table 2). In terms of remission inducing and

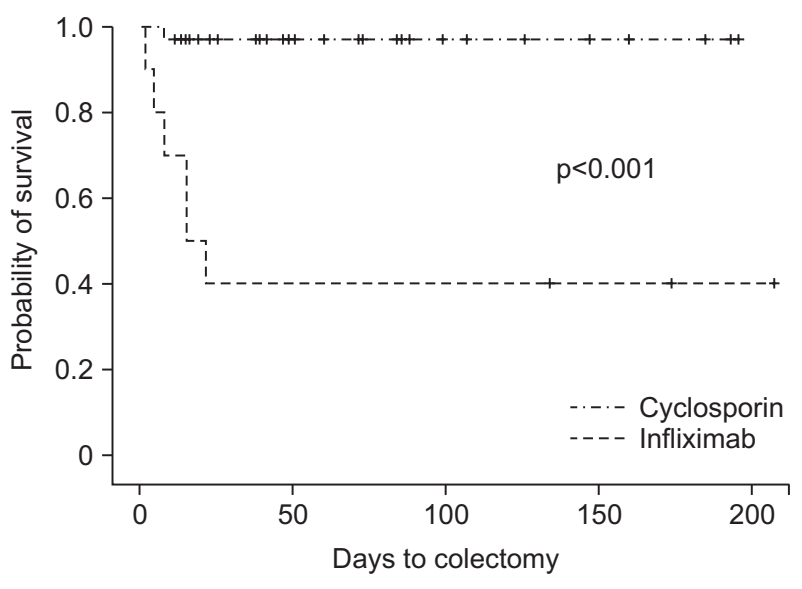

Fig. 1. Kaplan-Meier plots for colectomy-free survival according to the drug for rescue therapy.

maintenance rates, clinical remission was achieved numerically more frequently in infliximab group, but that was not statistically significant between the two groups (30.0\% vs $45.5 \%$, $\mathrm{p}=0.485$ ). Moreover, remission maintenance rates at 12 months were also not significantly different between the two groups (20.0\% vs $48.5 \%, p=0.153)$. At the end of the follow-up, the colectomy had been performed in six of 10 patients and one of 33 patients in the cyclosporine and the infliximab groups, respectively (cyclosporine vs infliximab, 60\% vs 3\%; $\mathrm{p}<0.001$ ). In six cases of patients who underwent colectomy after the treatment with cyclosporine, the median time to colectomy was 11.3 months with range of 1.7 to 21.3 months. The one patient who underwent colectomy after being treated with infliximab, the colectomy was done at 7.6 months after the treatment. The cumulative colectomy rates are shown in the Kaplan-Meier plot in Fig. 1. Colectomy-free survival in the infliximab group was superior to that in the cyclosporine group ( $p<0.001)$. In contrast to the result of univariate analysis, Cox proportional hazard 
Table 3. Cox Proportional Hazard Model for Colectomy

\begin{tabular}{lcc}
\multicolumn{1}{c}{ Parameter } & HR $(95 \% \mathrm{CI})$ & p-value \\
\hline Disease extent & 1 & 0.936 \\
Nonextensive & $6.9 \times 10^{4}\left(<0.001-4.116 \times 10^{122}\right)$ & \\
Extensive & $0.329(0.044-2.482)$ & 0.281 \\
Azathioprine & 1 & \\
Drug for rescue therapy & $0.166(0.013-2.088)$ & 0.164 \\
Cyclosporine & & \\
Infliximab & 0.160
\end{tabular}

HR, hazard ratio; CI, confidence interval.
Table 4. Cox Proportional Hazard Model for Colectomy in Patients Treated with Either Cyclosporine Alone or Infliximab with Azathioprine

\begin{tabular}{lcc}
\hline \multicolumn{1}{c}{ Parameter } & HR $(95 \% \mathrm{CI})$ & $\mathrm{p}$-value \\
\hline Disease extent & 1 & 0.955 \\
$\quad$ Nonextensive & $6.4 \times 10^{4}\left(<0.001-1.517 \times 10^{172}\right)$ & \\
$\quad$ Extensive & 1 & 0.017 \\
Drug for rescue therapy & & \\
$\quad \begin{array}{l}\text { Cyclosporine only } \\
\text { Infliximab with azathioprine }\end{array}$ & $0.073(0.008-0.629)$ & \\
\hline HR, hazard ratio; CI, confidence interval. &
\end{tabular}

Table 5. Secondary End-Points

\begin{tabular}{lccc}
\hline \multicolumn{1}{c}{ Parameter } & Cyclosporine $(\mathrm{n}=10)$ & Infliximab (n=33) & $\mathrm{p}$-value \\
\hline Length of hospital stay after rescue therapy, day & $41.8(0-94)$ & $6.6(0-38)$ & $<0.001$ \\
Patients on steroids at 12 months & 0 & $5(15.2)$ & 0.190 \\
Serious adverse events & $1(10.0)$ & $1(3.0)$ & 0.359 \\
\hline
\end{tabular}

Data are presented as median (range) or number (\%).

model showed that the drug for rescue therapy was not related to the colectomy-free survival (hazard ratio [HR] of infliximab compared to cyclosporine, 0.166; 95\% confidence interval [CI], 0.013 to 2.088) (Table 3). In addition, we proposed another Cox proportional hazard model for colectomy in patients treated with either cyclosporine only or infliximab with azathioprine, because most patients who received azathioprine belonged to the infliximab group (28 of 32 patients). Table 4 shows infliximab with azathioprine for rescue therapy was superior to cyclosporine only for preventing colectomy (HR of infliximab with azathioprine compared to cyclosporine only, 0.073; 95\% CI, 0.008 to 0.629 ).

Table 5 summarizes the results for the secondary end-points studied. Length of hospital stay after rescue therapy was shorter in the infliximab group $(p<0.001)$. The rates of corticosteroid dependence after 12 months did not differ between the two groups ( $p=0.190)$. The rates of adverse events also did not differ between the two groups ( $p=0.359)$. Pancytopenia was observed in one patient from each group. No serious operation-related complication occurred in both groups.

\section{DISCUSSION}

Corticosteroids are the mainstay of therapy for severe ulcerative colitis. ${ }^{21-23}$ However, at least one third of patients fail to respond to this treatment and ultimately undergo colectomy. ${ }^{22,24}$ In this population, rescue therapy with cyclosporine or infliximab has been used in recent years in an effort to decrease the rates of colectomy. ${ }^{18}$ However, there are theoretical and practical arguments regarding the use of infliximab versus cyclosporine. ${ }^{12}$ In one retrospective study in Korea, corticosteroids and cyclo- sporine combination therapy had no additional benefit over prolonged corticosteroids therapy alone. ${ }^{25}$

This study showed that colectomy-free survival was not affected by selection of drugs, infliximab versus cyclosporine, as a rescue therapy in patients with severe corticosteroid-refractory UC. This result was similar to the European hospital study. A randomized controlled trial which compared cyclosporine with infliximab in intravenous corticosteroid-resistant UC as a salvage therapy was previously performed at 27 European hospitals between June 1, 2007, and August 31, 2010. ${ }^{17}$ One hundred fifteen patients with acute severe steroid-refractory UC were recruited to receive either $2 \mathrm{mg} / \mathrm{kg}$ daily intravenous cyclosporine followed by an oral formulation ( $4 \mathrm{mg} / \mathrm{kg}$ daily) for 3 months, or infliximab (5 mg/kg) at weeks 0,2 , and 6 . Clinical responses at day 7 and steroid-free remission at day 98 were comparable between the groups (about 60\%), with no differences in terms of colectomy rates or adverse events. ${ }^{17}$ An early response (7 days) to either therapy exceeded $80 \% .^{17}$

In our study, when intravenous cyclosporine was successful at inducing remission, it was switched to oral azathioprine as described in other studies. ${ }^{26}$ In the literature, oral cyclosporine was reported to fail in maintaining remission for longer periods. As an alternative to oral cyclosporine, tacrolimus has also been successfully used in previous studies. ${ }^{27,28}$

In our study, however, infliximab seemed to be more effective than cyclosporine in terms with colectomy rate in the univariate analysis. At 12 months, the rate of colectomy was 30\% and $3 \%$ in the cyclosporine and the infliximab groups, respectively $(p=0.034)$. The difference between the results of univariate and multivariate analysis may be the higher frequency of the use of azathioprine in the infliximab group than in the cyclosporine 
group. In the subgroup analysis of our study, infliximab with azathioprine was superior to cyclosporine only for preventing colectomy.

Our data, which was colectomy rate of $60 \%$ in the cyclosporine group, are in agreement with the observational data reported by Leuven, which showed that $88 \%$ of patients with initial cyclosporine-induced remission underwent colectomy by the end of the follow-up period (7 years). ${ }^{29}$ However, in infliximabtreated patients, our results differed from those reported by the Swedish trial at 3 and 36 months, ${ }^{30,31}$ where the colectomy rates in the infliximab group were $29 \%$ and $50 \%$, respectively. These differences can be partially explained by the dose of infliximab. There was a report that showed data on colectomy rates at 12 months from the ACT I and II trials, which involved a multiple infusion regimen. ${ }^{32}$ These trials reported a lower colectomy rate of $10 \%$ for infliximab versus $17 \%$ for placebo. ${ }^{32}$ An analysis of the time to response in these two trials revealed that 63\% of the total $74 \%$ who fully responded to the three infusions did so after the first dose, which increased to $86 \%$ of the total responders by the second dose. ${ }^{32}$ In our study, the patients in the infliximab group received infusions every 8 weeks. It is well known that the number of infliximab infusions affects outcomes, with higher early colectomy rates in those receiving a single infusion compared with those receiving two or more infusions. ${ }^{12,13,33,34}$

In a Korean multicenter retrospective study, infliximab was effective and safe in the treatment of active UC. The rates of clinical responses and remission were $87 \%$ and $45 \%$ at week 8 . That study was the first to show the efficacy of infliximab in Asian UC patients, and the results were superior to the previous Western studies. The study, however, showed the efficacy of infliximab without group comparison. Our study has the merit that the first study on comparison of efficacy of rescue therapies in Korean patients with severe corticosteroid-refractory UC. In addition, it showed that the efficacy for preventing colectomy did not differ between the two drugs, infliximab versus cyclosporine. We think, however, this result do not imply that cyclosporine is as useful as infliximab. Although incidence of severe adverse events did not differ between both groups in the study, generally cyclosporine has more risks of adverse events compared to infliximab. ${ }^{2,35}$ According to our subgroup analysis in patients treated with either cyclosporine only or infliximab with azathioprine, infliximab with azathioprine was more effective for preventing colectomy than cyclosporine without azathioprine. Therefore, infliximab with azathioprine could be preferentially considered rather than cyclosporine alone in the clinical setting of severe steroid refractory UC.

The limitations of this study include the retrospective nature of the study design and the comparisons that were made between the two groups enrolled during different periods of time. This type of limitation should be overcome by well-designed prospective studies. Moreover, the small sample size limits the results as there were large ranges in baseline patient character- istics, making it difficult to precisely compare the two populations. However, the data obtained from this study are quite reliable considering the fact that the characteristics of the two groups were comparable using our internal database system for medical records. The main outcome in this study was colectomy rate, which was not biased by subjective evaluation. Additionally, the clinical management of severe colitis has not changed within the last two decades.

In conclusion, there was no difference between infliximab and cyclosporine with respect to prevent colectomy in severe corticosteroid-refractory UC patients. Infliximab with azathioprine, however, may be more effective than cyclosporine only for preventing colectomy.

\section{CONFLICTS OF INTEREST}

No potential conflict of interest relevant to this article was reported.

\section{ACKNOWLEDGEMENTS}

This research was supported by the Basic Science Research Program through the National Research Foundation of Korea (NRF) funded by the Ministry of Education, Science and Technology (grant number: 2011-0025131) and the NRF funded by the Ministry of Science, ICT \& Future Planning (MSIP) (NRF2013R1A2A2A01067123).

\section{REFERENCES}

1. Gracie DJ, Ford AC. Evidence-based management of ulcerative colitis. Minerva Gastroenterol Dietol 2012;58:87-99.

2. Dean KE, Hikaka J, Huakau JT, Walmsley RS. Infliximab or cyclosporine for acute severe ulcerative colitis: a retrospective analysis. J Gastroenterol Hepatol 2012;27:487-492.

3. Dinesen LC, Walsh AJ, Protic MN, et al. The pattern and outcome of acute severe colitis. J Crohns Colitis 2010;4:431-437.

4. Truelove SC, Witts LJ. Cortisone in ulcerative colitis: final report on a therapeutic trial. Br Med J 1955;2:1041-1048.

5. Truelove SC, Jewell DP. Intensive intravenous regimen for severe attacks of ulcerative colitis. Lancet 1974;1:1067-1070.

6. Kim DH, Cheon JH, Park JJ, et al. Clinical outcomes and predictive factors for response after the first course of corticosteroid therapy in patients with Crohn's disease. Gut Liver 2013;7:58-65.

7. Gearry RB, Falvey JD. Rescue therapy for steroid refractory acute severe ulcerative colitis: more choice, better outcomes? J Gastroenterol Hepatol 2012;27:417-418.

8. Kaur M, Targan SR. Ulcerative colitis: steroid-refractory ulcerative colitis-ciclosporin or infliximab? Nat Rev Gastroenterol Hepatol 2013;10:8-9.

9. Järnerot G, Rolny P, Sandberg-Gertzén H. Intensive intravenous treatment of ulcerative colitis. Gastroenterology 1985;89:1005- 
1013.

10. Lichtiger S, Present DH, Kornbluth A, et al. Cyclosporine in severe ulcerative colitis refractory to steroid therapy. N Engl J Med 1994;330:1841-1845.

11. Levesque BG, Sandborn WJ. Infliximab versus ciclosporin in severe ulcerative colitis. Lancet 2012;380:1887-1888.

12. Rolny P, Vatn M. Cyclosporine in patients with severe steroid refractory ulcerative colitis in the era of infliximab: review article. Scand J Gastroenterol 2013;48:131-135.

13. Singh S, Loftus EV Jr. Management of severe steroid-refractory ulcerative colitis: cyclosporine or infliximab? Gastroenterology 2013;144:1138-1140.

14. Arts J, D'Haens G, Zeegers M, et al. Long-term outcome of treatment with intravenous cyclosporin in patients with severe ulcerative colitis. Inflamm Bowel Dis 2004;10:73-78.

15. Rutgeerts P, Sandborn WJ, Feagan BG, et al. Infliximab for induction and maintenance therapy for ulcerative colitis. N Engl J Med 2005;353:2462-2476.

16. Järnerot G, Hertervig E, Friis-Liby I, et al. Infliximab as rescue therapy in severe to moderately severe ulcerative colitis: a randomized, placebo-controlled study. Gastroenterology 2005;128:1805-1811.

17. Laharie D, Bourreille A, Branche J, et al. Ciclosporin versus infliximab in patients with severe ulcerative colitis refractory to intravenous steroids: a parallel, open-label randomised controlled trial. Lancet 2012;380:1909-1915.

18. Lee KM, Jeen YT, Cho JY, et al. Efficacy, safety, and predictors of response to infliximab therapy for ulcerative colitis: a Korean multicenter retrospective study. J Gastroenterol Hepatol 2013;28:1829-1833.

19. González-Altamirano J, Paz-Delgadillo J, Bosques-Padilla F. Advances in the diagnosis, pathophysiology and treatment of chronic non-specific ulcerative colitis. Rev Gastroenterol Mex 2013;78 Suppl 1:65-67.

20. Mocciaro F, Renna S, Orlando A, et al. Cyclosporine or infliximab as rescue therapy in severe refractory ulcerative colitis: early and long-term data from a retrospective observational study. J Crohns Colitis 2012;6:681-686.

21. Kim ES, Kim WH. Inflammatory bowel disease in Korea: epidemiological, genomic, clinical, and therapeutic characteristics. Gut Liver 2010;4:1-14.

22. Lee JH, Cheon JH, Kim ES, et al. The prevalence and clinical significance of perinuclear anti-neutrophil cytoplasmic antibody in Korean patients with ulcerative colitis. Dig Dis Sci 2010;55:14061412 .
23. Lee JH, Cheon JH, Moon CM, et al. Do patients with ulcerative colitis diagnosed at a young age have more severe disease activity than patients diagnosed when older? Digestion 2010;81:237-243.

24. Lee HJ, Jung ES, Lee JH, et al. Long-term clinical outcomes and factors predictive of relapse after 5-aminosalicylate or sulfasalazine therapy in patients with mild-to-moderate ulcerative colitis. Hepatogastroenterology 2012;59:1415-1420.

25. Chung GE, Cheon JH, Lee JY, et al. Efficacy of combination of intravenous cyclosporin A and steroid therapy versus prolonged intravenous steroid therapy alone in patients with severe ulcerative colitis refractory to initial intravenous steroid therapy. Korean J Gastroenterol 2006;48:263-268.

26. Cohen RD, Stein R, Hanauer SB. Intravenous cyclosporin in ulcerative colitis: a five-year experience. Am J Gastroenterol 1999;94:1587-1592.

27. Ogata $\mathrm{H}$, Matsui $\mathrm{T}$, Nakamura $\mathrm{M}$, et al. A randomised dose finding study of oral tacrolimus (FK506) therapy in refractory ulcerative colitis. Gut 2006;55:1255-1262.

28. Baumgart DC, Pintoffl JP, Sturm A, Wiedenmann B, Dignass AU. Tacrolimus is safe and effective in patients with severe steroidrefractory or steroid-dependent inflammatory bowel disease: a long-term follow-up. Am J Gastroenterol 2006;101:1048-1056.

29. Moskovitz DN, Van Assche G, Maenhout B, et al. Incidence of colectomy during long-term follow-up after cyclosporine-induced remission of severe ulcerative colitis. Clin Gastroenterol Hepatol 2006;4:760-765.

30. Sands BE, Tremaine WJ, Sandborn WJ, et al. Infliximab in the treatment of severe, steroid-refractory ulcerative colitis: a pilot study. Inflamm Bowel Dis 2001;7:83-88.

31. Travis S, Satsangi J, Lémann M. Predicting the need for colectomy in severe ulcerative colitis: a critical appraisal of clinical parameters and currently available biomarkers. Gut 2011;60:3-9.

32. Sandborn WJ, Rutgeerts P, Feagan BG, et al. Colectomy rate comparison after treatment of ulcerative colitis with placebo or infliximab. Gastroenterology 2009;137:1250-1260.

33. Farkas K, Lakatos PL, Nagy F, et al. Predictors of relapse in patients with ulcerative colitis in remission after one-year of infliximab therapy. Scand J Gastroenterol 2013;48:1394-1398.

34. Löwenberg M, de Boer NK, Dewint P, Hoentjen F. Treatment of patients with severe glucocorticoid-refractory ulcerative colitis: cyclosporine or infliximab? Ned Tijdschr Geneeskd 2013;157:A6130.

35. Croft A, Walsh A, Doecke J, Cooley R, Howlett M, Radford-Smith G. Outcomes of salvage therapy for steroid-refractory acute severe ulcerative colitis: ciclosporin vs. infliximab. Aliment Pharmacol Ther 2013;38:294-302. 